\title{
Percutaneous Bioelectric Current Stimulation for Chronic Cluster Headache - A Possible Transformative Approach to Cluster Headache
}

This article was published in the following Dove Press journal: Journal of Pain Research

\author{
Albrecht Molsberger' \\ Colin D McCaig ${ }^{2}$ \\ 'Department of Orthopedics, Ruhr- \\ University Bochum, Germany, Clinic for \\ Headache, Pain and Locomotive \\ Disorders, Duesseldorf, Germany; \\ ${ }^{2}$ Institute of Medical Sciences, University \\ of Aberdeen, Aberdeen AB25 2ZD, \\ Scotland
}

Background: Cluster headache $(\mathrm{CH})$ is considered to be a catastrophic disease presenting the most severe human pain condition. Available pharmacological treatments are hampered by unwanted side effects, and there is an urgent need for non-pharmacological treatment alternatives. We present a novel therapeutic approach for chronic $\mathrm{CH}$, having evolved from an episodic $\mathrm{CH}$, using a non-invasive percutaneous bioelectric current stimulation (PBCS), which generates static electric fields in the range of the naturally occurring electric potentials. Patients and Methods: This study employed a retrospective data analysis of 20 cases of chronic cluster headache $(\mathrm{CCH})$ patients, four of those having had cluster-related surgery (SPG, ONS). All patients were treated with PBCS between 2014 and 2018. Data of these patients were analyzed with respect to frequency of $\mathrm{CH}$ attacks and triptan application and followed up for one (20 cases) or two (12 cases) years.

Results: Four weeks after the first PBCS treatment, cluster headache attacks were reduced from 2.8 to 1.7 per day and triptan application decreased from 2.5 to 1.5 times/day. Six nonresponders, 4 of which had pre-CH surgery, did not show any reaction to PBCS, while 14 responders improved within 4 weeks from 2.2 to 0.7 attacks/day and 2.0 to 0.4 triptan applications/day. A $50 \%$ or greater reduction of attack frequency was observed in 10 patients after 4 weeks and in 11 patients after 12 weeks. One year after the first treatment, 13/20 patients experienced a reduction of attack frequency of $50 \%$ or more, while remarkably 10 patients were completely free of attack. After 2 years, 8 of 12 patients experienced a reduction of attack frequency of $50 \%$ or more and 7 of those were completely symptomfree. No serious adverse effects were observed.

Conclusion: $\mathrm{PBCS}$ is a promising transformative treatment approach for $\mathrm{CCH}$ patients. Drug consumption was reduced significantly, and the $\mathrm{CCH}$ may revert back to an episodic cluster headache with increasingly long times of remission. Responders can be clearly differentiated from non-responders. The data support the need for randomized controlled trials.

Keywords: chronic cluster headache, bioelectric current, pain, direct current

\section{Introduction}

Cluster headache $(\mathrm{CH})$ is regarded as a rare, but catastrophic, most severe pain disorder. The prevalence of $\mathrm{CH}$ is estimated in a wide range between 7 and 160 patients per 100.000 (1:1000 as a rule of thumb, so in Germany 5600 to 95,200, and in the US about 500.000 patients are affected). ${ }^{1,2}$ It is characterized by unilateral headache attacks lasting 15-180 mins, which occur on average 2-3 times a day. The pain is localized around the eye and can radiate to the upper jaw, temples, and
Correspondence: Albrecht Molsberger Fax +492118668818

Email albrechtmolsberger@mac.com 
Long lasting remissions

Patient 1

${ }_{6}^{8} \longrightarrow$

2 Tllith

6
5
3
2
1
0

Patient 3

6
5
3
2
1
0
$3^{8}+40,4$

Long lasting remissions with easier recurrent episodes

Patient 4

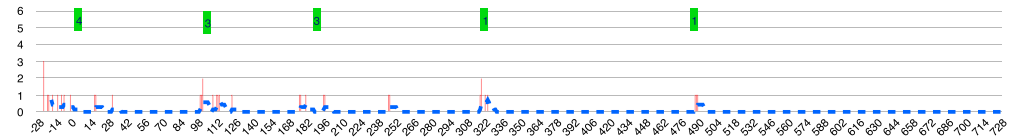

Patient 5

$6-1 \quad 3 \quad 4$
$5-1+4$

?

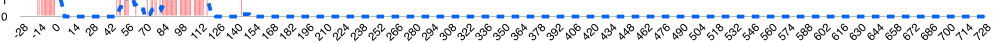

Patient 6

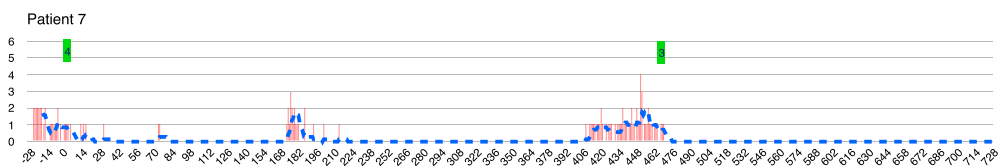

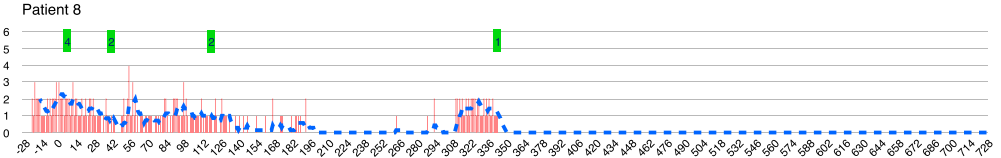
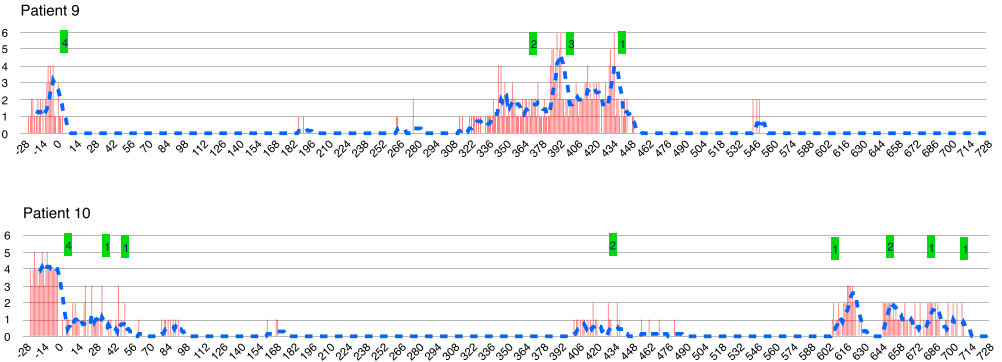

Figure I Continued. 
Long lasting remissions with more severe recurrent episodes
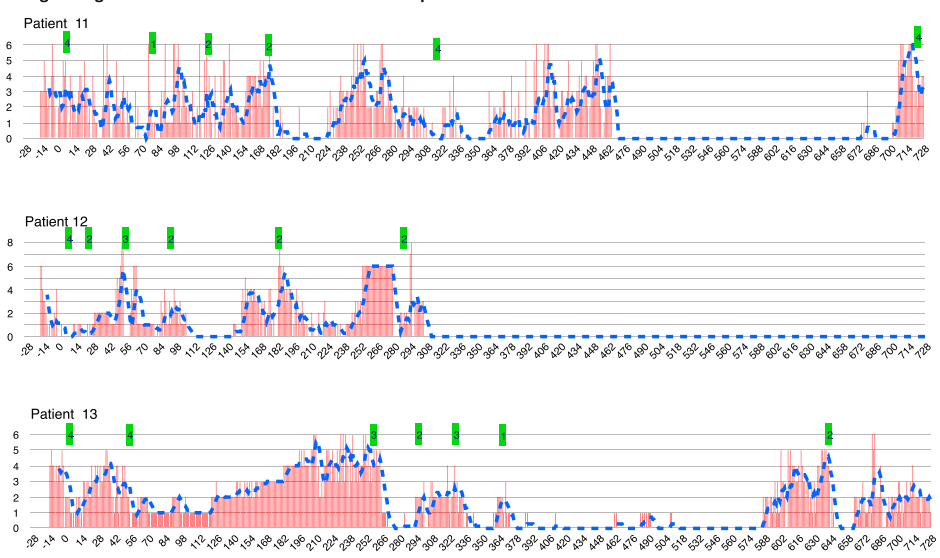

Minor, not lasting improvement

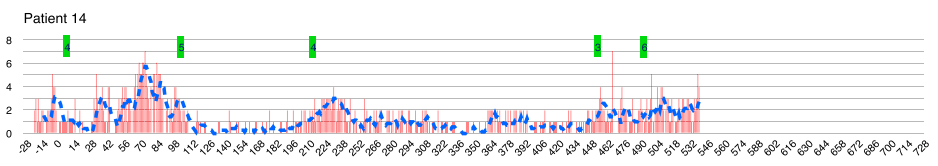

Complete Non-Responders

Patient 15

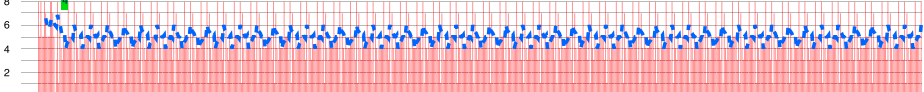

0
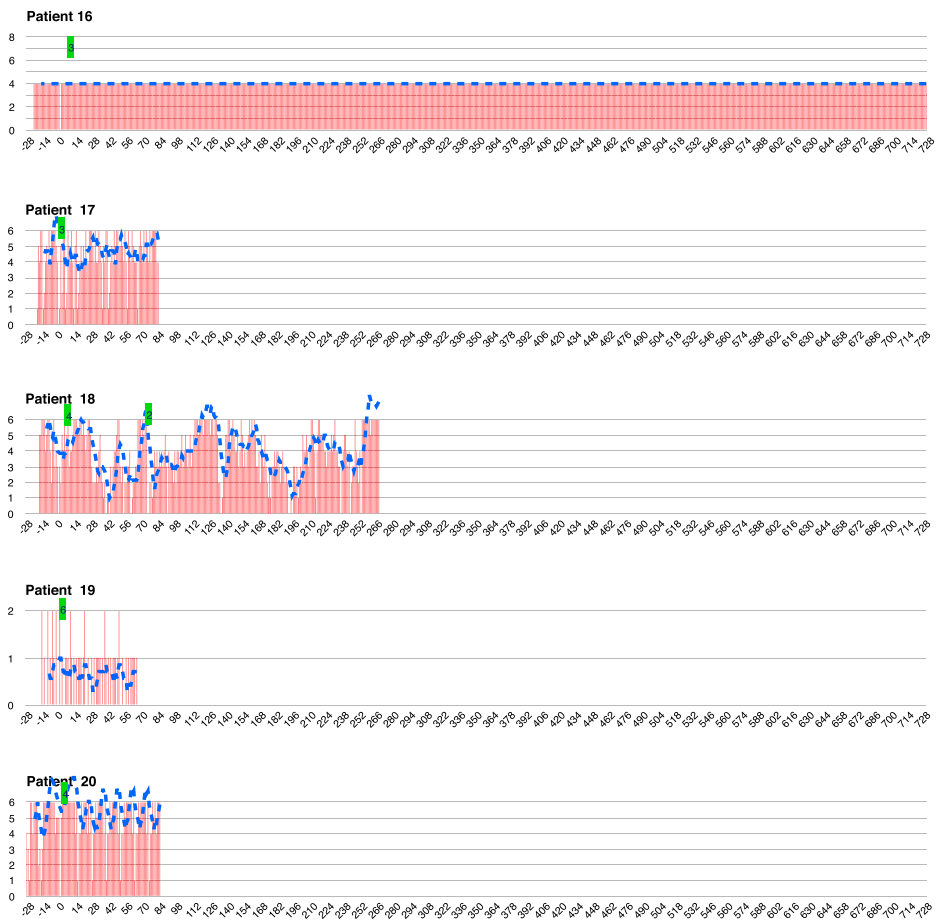

Figure I X axis $=$ no. of days of follow up; $y$ axis $=$ no. of attacks a day. Red bars $=$ no. of attacks a day; blue dotted line $=7-$ day moving average. Green label $=$ no. of PBCS treatments at this time point. Patient 15-20 discontinued headache diary, they were followed up by telephone interview and last value carried over method was used to impute missing data. 
forehead and back of the head. The pain is usually perceived as burning and stabbing and has a devastating character. ${ }^{1,2}$ In the episodic form of $\mathrm{CH}$, headache attacks are more frequent in spring and autumn, with pain-free intervals in between. About $10-15 \%$ of $\mathrm{CH}$ patients suffer from a chronic form $(\mathrm{CCH})$ with attacks occurring for 1 year or longer without remission or with remission periods lasting less than 3 months. Chronic forms are further subdivided as a primary $\mathrm{CCH}$ which is chronic from the very first onset of the disease and an "evolved" form, which evolves from an episodic form. ${ }^{3}$ Acute therapy consists of the administration of oxygen inhalation and triptan nasal sprays or injections. Verapamil, topiramate and lithium are usually given as a prophylactic treatment. ${ }^{4}$ Due to the intensive treatment, the annual costs are between 5.000 and 20.000 Euros per patient. ${ }^{2}$

Available pharmacological treatments are hampered by unwanted side effects and there is an urgent need for new non-pharmacological treatment alternatives ${ }^{5-9}$ There are interesting new developments in electronic stimulation methods for neuromodulation. These include occipital nerve stimulation (ONS) (evidence level IV), microstimulation of the ganglion sphenopalatine (SPG) and noninvasive vagus stimulation (nVNS) (the latter two with evidence Level 2). ${ }^{10,11}$ All these forms of stimulation have in common that they work with frequencies, pulse widths and currents in the milliampere range, as they have been used for decades in transcutaneous nerve stimulation. The common rationale is to interrupt the afferent pathway by overriding the electrical capacity of nerves to generate further action potentials. In contrast to these neurostimulator devices, we present here a novel approach that uses percutaneous bioelectric direct current stimulation (PBCS). This electrical stimulation modulates tissue-specific small electric fields like transepithelial potentials thus interacting with charged ions and peptides. PBCS was originally developed for locomotive problems; however, incidentally we observed a clinical effect in Cluster headache. In the following, we present a retrospective analysis of $\mathrm{CCH}$ patients having been treated with remarkable success by this new treatment. ${ }^{12}$

\section{Materials and Methods}

\section{Patient Characteristics}

During the preceding 4 years, patients with episodic and chronic cluster headache $(\mathrm{CCH})$ were treated with percutaneous bioelectric current stimulation (PBCS) in our pain clinic. ${ }^{12}$ For this study we performed a retrospective data analysis of all patients meeting the following inclusion criteria: informed consent; chronic cluster headache, which had evolved from episodic cluster headache; at least 11 months of continues $\mathrm{CH}$ prior to PBCS treatment; a minimum of 3 attacks per week, triptan and/or oxygen being effective for acute treatment. Patients were not actively recruited; however, they reported for therapy after they heard in German social media $(\mathrm{CH}$ patient forums, self-help groups) from other patients about PBCS. All patients were asked to fill in a headache diary beginning at least 2 weeks before the first treatment and continuing throughout the treatment and follow up time up to 2 years. Typical $\mathrm{CH}$ characteristics had to be reported, including attack frequency, pain severity on a numeric rating scale (NRS) from $0=$ no pain at all to $10=$ unbearable pain, duration of attack and medication consumption. Data were collected during clinical visits and by email and telephone interviews.

\section{Treatment Characteristics and Outcome \\ Assessment}

The PBCS treatment system has been approved and certified for pain treatment by a notified body of the European Commission (CE0482). A mobile, hand-held electroceutical smart device, based on a microcontroller, an analog frontend and a battery generates DC electric fields (EFs) mimicking and modulating those electrical signals, which are observed in inflammation, wound healing and tissue regeneration. A large body of evidence exists, showing that these small DC EFs provide pivotal directional cues for the migration of inflammatory cells (monocytes, macrophages), epithelial cells, fibroblasts and nerve cells ${ }^{13-17}$ Extensive technical details as well as the physiological rational of this novel treatment has been published recently. ${ }^{12}$

Patients received PBCS treatments on a weekly or biweekly basis. Insertion points usually were located within these topographical areas: below the maxilla, $1-4 \mathrm{~cm}$ lateral to the ala of the nose; the medial end of the eyebrow; the middle of eyebrow; on the forehead $1 \mathrm{~cm}$ above the middle of the eyebrow; at the temporal region; at the vertex $1-2 \mathrm{~cm}$ lateral to the midline; the occipital region above the major occipital nerve. To precisely locate the points selected for stimulation, patients were asked to indicate the typical painful areas appearing during an attack, which were confirmed by applying pressure with a $6 \mathrm{~mm}$ thick rounded metal probe, similar to a "follow the 
pain" strategy as it is used for botulinum toxin injection or acupuncture in migraine. ${ }^{18,19}$ Then, an unsiliconised acupuncture needle $(0,25$ in diameter) was inserted carefully until the patient felt a sharp increase of pain. Secondly, the needle was redrawn for a few millimeters, just enough to avoid further eliciting pain. Depending on the size of the area to be treated up to 9 needle probes were inserted, usually 1 probe per square centimeter. In these cases, the depth of insertion was defined by the location of the area to be treated and ranged from $0.5 \mathrm{~cm}$ (eg, temporal region) to $4 \mathrm{~cm}$ (below the maxilla and arcus zygomaticus). A conductive skin pad was placed on the ipsilateral upper arm above the musculus deltoideus. The anodal output of the digital stimulator device was then connected to the skin pad and the cathodal output to the needle probes. Finally, an undulating direct current in the range of 15-100 $\mu \mathrm{A}$ per needle was applied for $30 \mathrm{mins}$.

To assess the effectiveness of PBCS we measured reductions in the number of attacks and in the consumption of triptans per day in comparison to baseline. Adverse events were collected from patients' notes. As a retrospective outcome chart analysis of a certified medical device treatment having been used for individual curative treatments, the German medical association stated that an ethics committee approval was not required.

\section{Results}

Twenty patients, 5 female, 15 male, mean age 45 years, suffering from $\mathrm{CCH}$, all evolved from $\mathrm{ECH}$, were treated with PBCS. Four of the $\mathrm{CCH}$ patients had a history of several cluster-related surgeries including the implantation of an electrical stimulator of the ganglion sphenopalatine (SPG stimulation) and/or of the nervus occipitalis major (ONS); one patient had received six operations at the corresponding nasal sinuses. At the time of the start of PBCS therapy, none of the stimulators was being used either because they had not shown any relevant effect on the patient in the past (SPG), or the effect has vanished over time (ONS). Since this is a first observational study, the following description includes those pre-operated patients.

The mean duration of $\mathrm{CH}$ was 13 years, first as an episodic form, then during the last 6 years changing into a chronic $\mathrm{CH}$ form. In 10 patients the pain was on the right and in 8 patients on the left side; in two patients the pain changed from one side to the other. All patients had a history of the typical acute (triptans, inhalation of oxygen) and prophylactic treatments (verapamil, lithium, topiramate). The diagnosis was confirmed by at least one neurologist, six patients having been treated in an ambulatory, six in a stationary specialized headache center and five in both. All patients had tried cortisone treatment without lasting effect. Before the beginning of the PBCS treatment, 11 patients took verapamil (mean daily dosage $585 \mathrm{mg}$ ), while nine patients had stopped the prophylactic verapamil treatment because of insufficient effect or unwanted side effects. Two patients only used oxygen for the acute treatment, two patients had replaced triptans because of unbearable side effects - one took metamizole for more than 10 years, the other Ergotamine for the last 15 years (individual patient data are presented in Table 1). With the above prophylactic treatment, the typical patient suffered from 2.8/2.3 (mean/median) attacks per day with a pain severity of $6.7 / 6.7$ and would take 2.5/2.3 triptans (nasal application or injections) as an acute treatment (all values at baseline, see Table 1). She/He received 3.9/4 PBCS treatments within 2 weeks. Four and (12) weeks after the first treatment, the number of attacks per day had reduced to $1.7 / 0.8(1.4 / 0.6)$, the pain severity to $3.1 / 2.2$ $(3.1 / 2.4)$ and the triptan intake to $1.5 / 0.3(1.4 / 0.4)$ (Table 1, Figure 2). A $50 \%$ or more reduction of pain frequency was observed in 10 patients after four and in 11 patients after 12 weeks of stimulation (Table 1).

All 20 patients could be followed up for 12 months. During this time span, 12 patients showed a recurrence of the attacks, which were stopped by one or more courses of PBCS treatment (Table 1, PBCS treatments, Figure 1). At 12 months follow up 13 patients experienced a reduction of attack frequency of $50 \%$ or more, 10 of those being symptom-free without any attacks. After 24 months eight patients experienced a reduction of attack frequency of $50 \%$ or more, 7 of those being symptom-free (Table 1, last two rows). No serious adverse effects were observed.

Of the six patients who showed no response to PBCS therapy, four belonged to the group of pre-operated patients and one other patient had developed $\mathrm{CH}$ after a suicide attempt with Herphonal. Overall, the non-responders compared to the responders had higher baseline values for attack rate (4.3 versus 2.2 (mean)), pain severity (7.6 versus 6.3 ) and triptan consumption (3.8 versus 2.0 ). In a stratified evaluation, in non-responders, there was no change in the number of attacks (4.3/4.2 (mean)) and triptan consumption (3.8/4.1 within the first 4 weeks. In the responder group, the number of attacks was reduced from 2.2 to 0.7 (mean) and the triptan consumption from 2.0 to 0.4 within the first 4 weeks. 


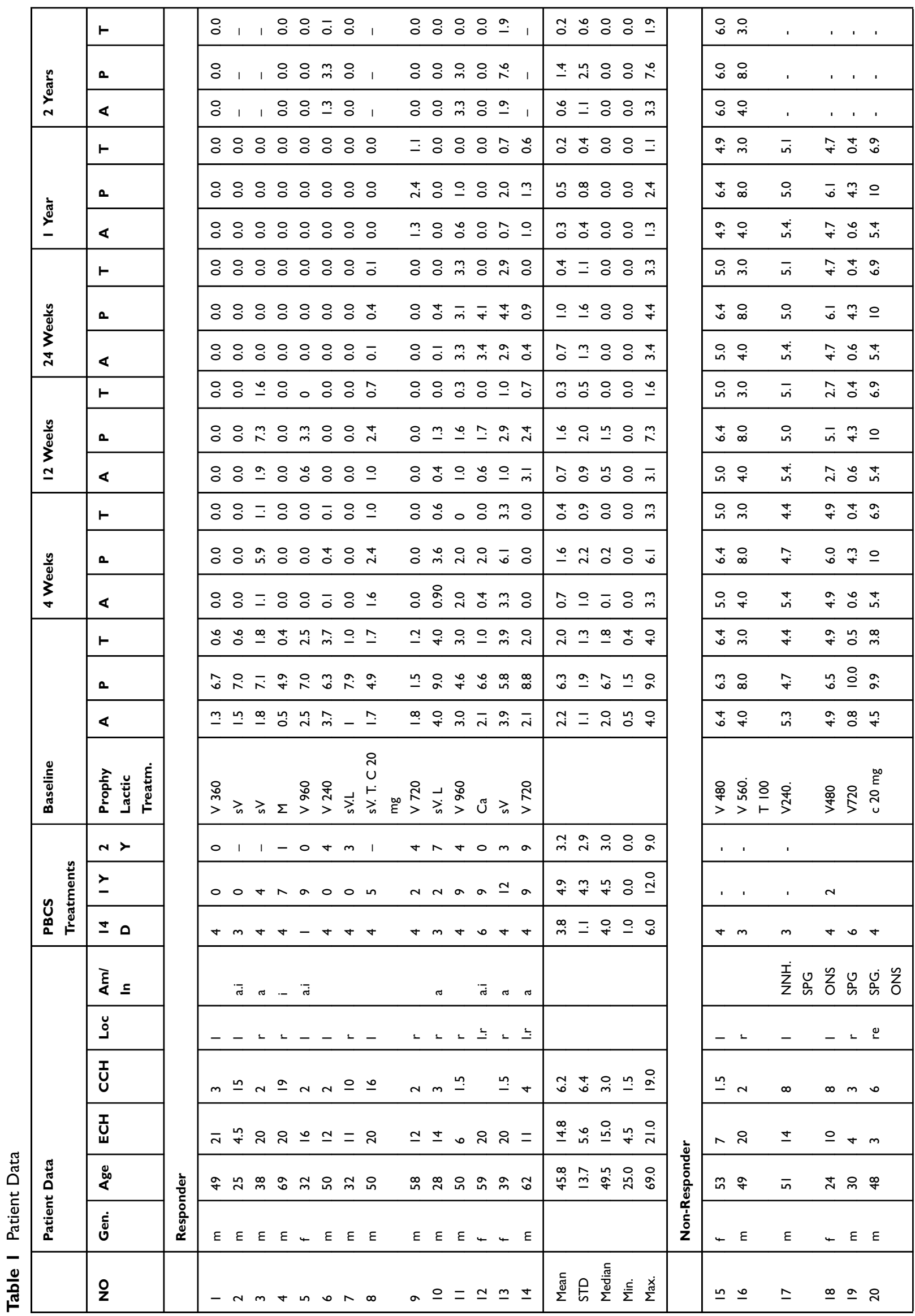




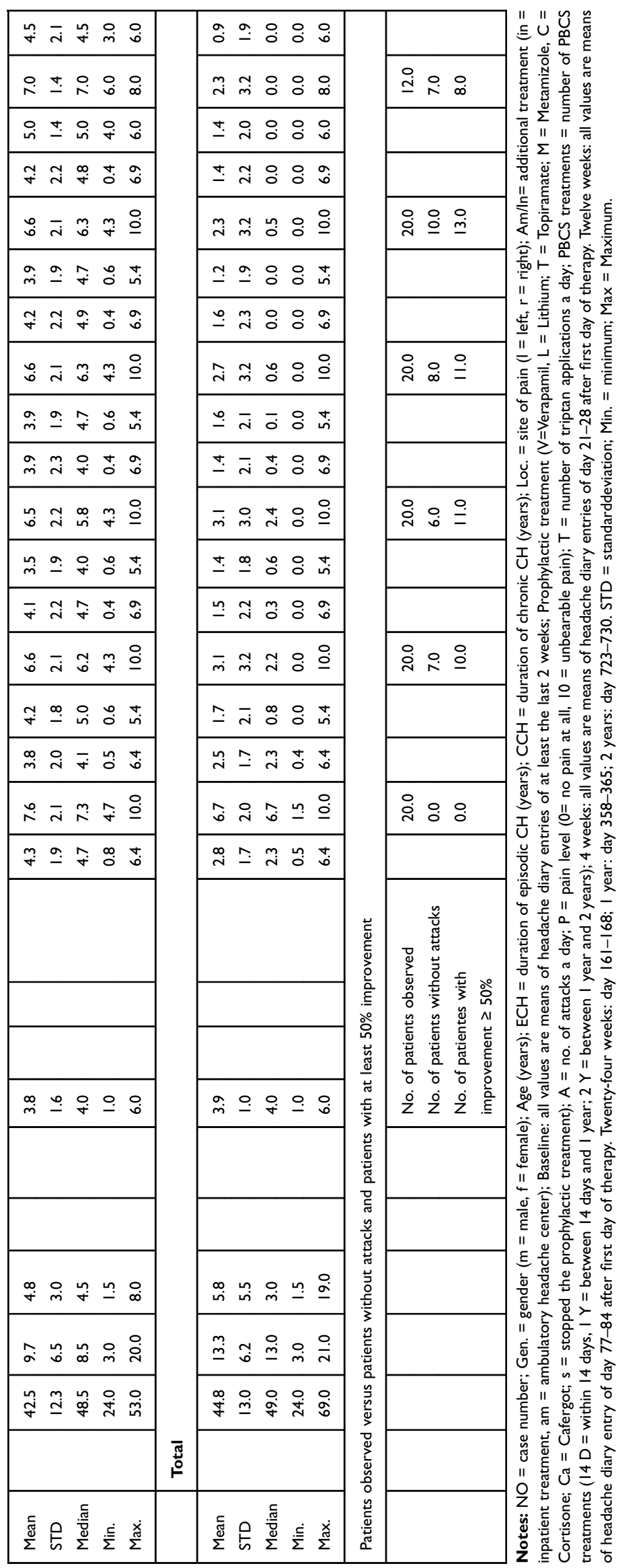



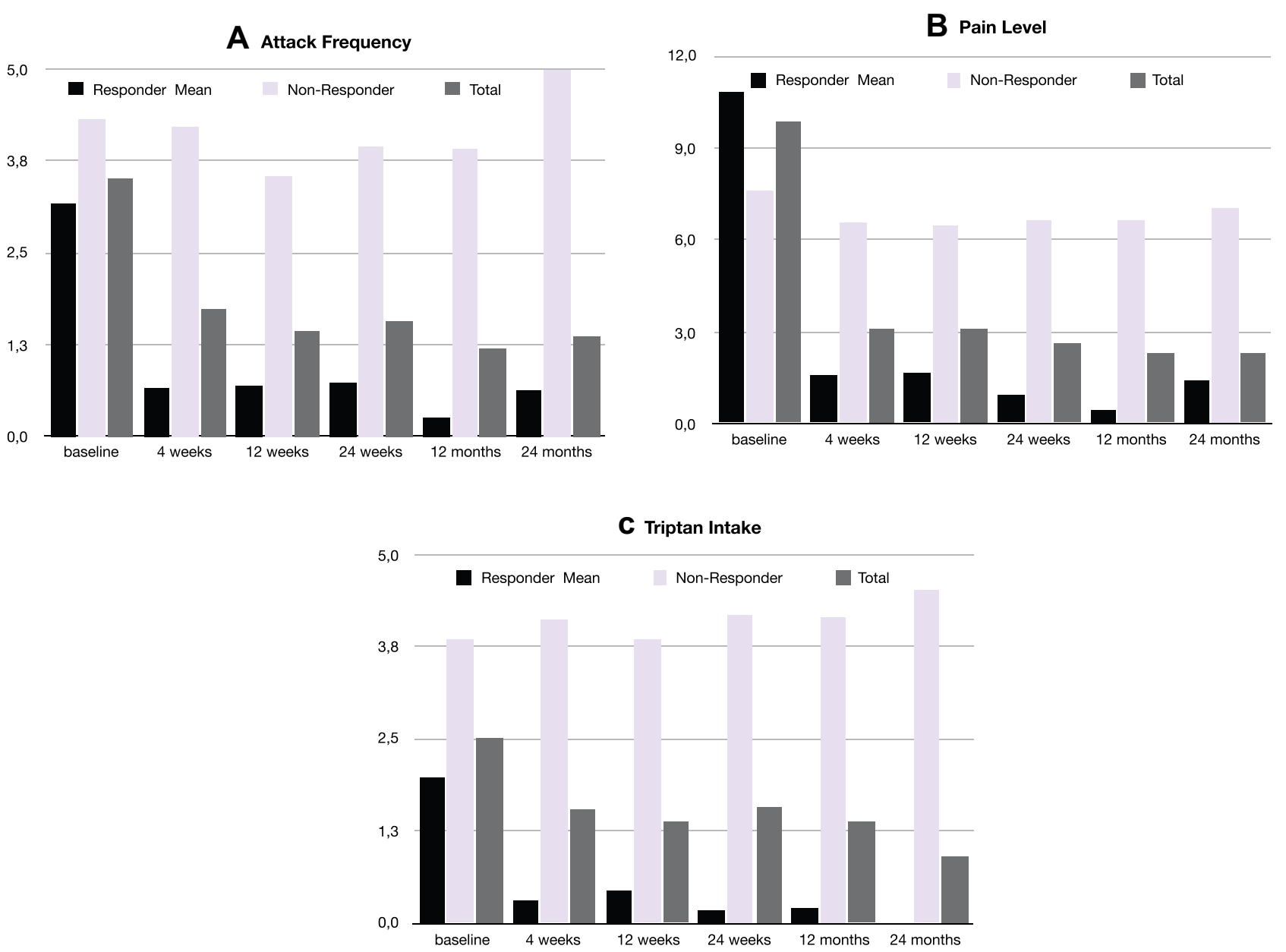

Figure 2 (A) Attack frequency, (B) pain level, (C) triptane intake. Mean values for all patients, responders and non-responders, respectively.

Below, we describe six patient cases (patient 2, 4. 7, $12,14,15)$ illustrating five typical patterns of reaction to the PBCS treatment (Figure 1). As supplementary material, four video testimonials (patient $7,10,11,12$ ) and patterns of all patients are provided. Video 2 also shows the device and demonstrates the treatment.

\section{No Reaction to PBCS - Complete Non- Responders}

\section{Cases 15-20}

\section{Example Case 15}

Fifty-three-year-old female patient with a history of 7 years of $\mathrm{CH}$, the last 1.5 years being chronic. $\mathrm{CH}$ started with an overdose of Herphonal immediately after an attempted suicide due to depression. The current therapy consisted of Sumatriptan 2 tablets daily, oxygen inhalation and Verapamil $480 \mathrm{mg}$ per day. Four PBCS therapies within 9 days showed no effect at all in terms of attack rate or frequency, so the therapy attempt was terminated.
In the following 12 months, further therapeutic trials with cortisone, lithium and topiramate were discontinued due to intolerance. Due to the severity of the disease, several applications for retirement have been submitted.

\section{Long-Lasting Remissions}

Cases I, 2, 3

Example Case 2: 25-year-old male patient with a history of 6 years of $\mathrm{CH}$, the last 1.5 years being chronic. In the past Verapamil $240 \mathrm{mg}$ had to be stopped because of bradycardia and attacks could have been stopped only with cortisone courses. Before the treatment he had suffered from 1.5 attacks per day, being treated with Triptan nasal application and oxygen inhalation. The patient received 3 PBCS treatments within 7 days. Among them, the number of attacks and pain intensity decreased slightly. However, without any further therapy 2 weeks after the last PBCS treatment, the attacks were completely suspended. The patient has now been symptom-free for more than 1 year, showing long-lasting remission. 


\section{Long-Lasting Remissions with Easier \\ Recurrent Episodes}

Cases 4, 5, 6, 7, 8, 9, 10

Example Case 4

Sixty-nine-year-old male patient with a history of 20 years of $\mathrm{CH}$, the last 19 years being chronic. Besides cluster attacks every other day, he had suffered from a $24 \mathrm{hr}$ concomitant level 4 pain homolateral beneath the eye in the region of the upper jaw. At the beginning he received 4 PBCS treatments with subsequent complete pain relief of the concomitant pain and only very rare attacks; three months later and after 6 months he needed two more treatment series with 3 sessions. During the second year, he needed only one treatment to stay completely free of pain.

\section{Example Case 7}

Thirty-two-year-old male patient with a history of 11 years of $\mathrm{CH}$, the last 10 years being chronic. In the beginning, verapamil and lithium helped well, but were discontinued due to decreasing effect and concentration problems. Before PBCS treatment began, the patient suffered from one attack per day of pain level 8, each of which he treated with triptan nasal sprays, since the beneficial effect of oxygen inhalations had decreased last year. The patient received 4 PBCS treatments within 11 days. After the third treatment attacks were reduced significantly for 6 months, followed by an episode of light attacks, for which the patient did not seek treatment. Another 7 months later, a second episode of attacks occurred, again with somewhat less pain. After 3 further PBCS treatments, the patient was symptom-free.

\section{Long-Lasting Remissions with More Severe Recurrent Episodes \\ Cases II, 12, 13 \\ Example Case 12}

This is an example of a lengthy and ultimately successful course of PBCS therapy in a 59-year-old patient with a history of 20 years of $\mathrm{CH}$, the last 10 years being chronic. In the first years, the cluster was successfully treated with oxygen, triptans and verapamil. With decreasing effect the therapy was changed to $2 \mathrm{mg}$ Cafergot daily, 15 years ago. Until 6 years ago she was completely pain-free; since then the Cafergot therapy lost effect and she again suffered 4 attacks a day. Twice the patient tried to have an ergotamine withdrawal under cortisone protection in specialized headache clinics. The attempt failed both times because the attacks increased unbearably in frequency and pain intensity even under cortisone protection and additionally an ergotamine withdrawal headache occurred. Due to the frustrating therapy results, the patient was ultimately advised to implant an SPG stimulator. When she introduced herself for PBCS therapy, she suffered from daily attacks of pain level 7. Within 1 week, 3 PBCS stimulations were performed, resulting in the complete suspension of cluster attacks. Afterwards, the ergotamine could be reduced to zero within 2 weeks under further 3 PBCS stimulations to treat the ergotamine withdrawal headache. Interestingly without ergotamine therapy, $\mathrm{CH}$ attacks occurred again, albeit with a 50\% reduction in frequency and painfulness. Another 3 treatments and a subsequent weeklong cortisone treatment with $60 \mathrm{mg}$ prednisolone showed a different effect than in the past and in combination with 2 further PBCS therapies the patient was again completely painless in the following 6 weeks. Two more exacerbations followed, which were treated with 2 PBCS therapies each. Since then, the patient has been completely symptom-free without taking any medication (13 months).

\section{Minor, Not Lasting Improvement Case 14}

Example: 62-year-old male patient with a history of 11 years of $\mathrm{CH}$. At first, the $\mathrm{CH}$ was right-sided, but years ago the location switched to the left side and has been chronic ever since. Until recently he took $720 \mathrm{mg}$ verapamil but this was no longer tolerated and he now treated every attack exclusively with triptan injections and oxygen. When he started the PBCS treatment he had two to four attacks at night, the main pain being located behind the left eye, radiating into the lower jaw. Already after the second PBCS therapy attack frequency was reduced to only one attack per night. Also, the pain decreased so that he did not need any acute medication anymore. After the third treatment. the $\mathrm{CH}$ changed to the right side. After three further treatments, he was completely pain-free for 3 months. Then, the $\mathrm{CH}$ started again. This time, after his previous positive experiences with the PBCS therapy, the patient was highly motivated to be treated again. Eighteen further treatments improved the frequency of attacks, also the intensity of pain, but overall the clinical result was not sufficiently satisfactory so that the treatment attempt was terminated without lasting success.

\section{Discussion}

In this retrospective analysis, with a considerably long follow up period of up to 2 years, we have presented clinical cases of severe $\mathrm{CCH}$, evolved from $\mathrm{ECH}$, with the majority having 
benefited to a great extent from the new PBCS treatment approach, many achieving prolonged remission from a disease, which until now has been regarded as the most painful and incurable pain problem. Acute use of triptan medication also was significantly reduced following PBCS. No severe adverse events were observed. Compared to SPG, ONS or nVNS, which are primarily used on demand for the treatment of acute attacks, our data suggest, that PBCS stimulation has a strong prophylactic, long-term effect. Whether a PBCS therapy is helpful for a patient is apparent already within the first 2 weeks after a maximum of 4 treatments, as the following comparison between responders and non-responders suggests.

Six of 20 patients showed no response to PBCS treatments. For clinical use and for future studies it would be important to identify such non-responders in advance. According to our data - despite all reservations due to the small number of patients - a $\mathrm{CH}$-related previous operation, a higher number of attacks and a higher consumption of triptan can be considered as prognostically unfavorable. This almost binary distinction between nonresponders and responders is reflected in the clear differences between all mean and median values, as well as in a separate analysis of both groups. Here it can be seen that the characteristic values of the non-responders do not change. In responders, on the other hand, within 4 weeks after the first treatment the number of attacks is reduced by more than $60 \%$ and the triptan consumption by $80 \%$.

Similar to other pharmacological (Botox injection, nasal Lidocaine application, occipital Cortisone injections) or electrical stimulations of the occipital nerve or the ganglion sphenopalatine the stimulating probes are placed precisely at the patient's painful areas of the three trigeminal nerves, and in the vicinity of the ganglion sphenopalatine and the nervus occipitalis major. ${ }^{4-9}$ In distinction to other electrical stimulations, which use high intensity and frequency to reduce afferent transduction of pain signals or use highintensity direct currents for transcranial stimulation, PBCS stimulation uses small direct currents in the range of those found naturally in the body, such as those driven across epithelial (and endothelial) tissues by the transepithelial (or endothelial) potential difference (TEP). ${ }^{6}$ In PBCS currents of between 15 and $100 \mu \mathrm{A}$ are applied for 30 mins and since human tissue has a resistance of around $500 \Omega$ these will correspond to voltages of around $7.5-50 \mathrm{mV}$. Over a distance of several $\mathrm{mm}$, such a voltage drop has profound effects on the behaviors of many cell types. These include fibroblasts, epithelial cells, nerve cells, macrophages and monocytes, which collectively are required to orchestrate the regeneration of wounds. Such small electrical signals regulate and direct cell migration, the release of neurotransmitters, cytokines and several growth factors, the positioning and quantity of surface receptors for such ligands and promote an antiinflammatory effect (buffering of acidosis and disintegration of charged pro-inflammatory peptides) ${ }^{13-17}$ PBCS is therefore likely to induce the release and accumulation of a complex cocktail of molecules in the perivascular spaces of sensory nerves.

How might relatively short bursts of small electrical signals alleviate $\mathrm{CH}$ ? One possibility could involve the electrical regulation of mast cells. Mast cell progenitors home in on and increase in both the skin and the adventitia around the trigeminal nerve during $\mathrm{CH}$ attacks. ${ }^{20,21}$ They are present in elevated numbers at electroacupuncture acupoints where they release histamine, adenosine and cytokines amongst a cocktail of molecules. $^{22}$ This mast cell degranulation is evident also in $\mathrm{CH}$. Chemoattraction involving CXCL2 gradients and CXCR2 receptors present in mast cell progenitors promotes further mast cell accumulation at inflammation sites, for example, during $\mathrm{CH}$. Interestingly electric fields (EF) enhance CXCR2 expression in vascular endothelial cells. ${ }^{23}$ Although a number of blood-derived cells show directed cell migration in response to small electrical fields (macrophages, monocytes, neutrophils and $\mathrm{T}$ cells ${ }^{24,25}$ ), whether mast cells are electrosensitive and might accumulate focally at stimulation sites, is not known but is testable. However, if the EFs established by PBCS needles directed mast cell migration and regulated cytokine secretion and receptor expression as occurs in many other cell types, a mast cell-based explanation of the remarkable efficacy of PBCS in alleviating $\mathrm{CH}$ may emerge. Why such short periods of electrical stimulation have such long-lasting effects in abolishing $\mathrm{CH}$ remains unclear.

Typically, the evolved chronic cluster develops from the episodic form, as the painless intervals between cluster attack times become shorter and shorter until the cluster finally exists for more than 9 months per year. Unlike primary $\mathrm{CCHs}$, evolved $\mathrm{CCHs}$ can also experience painfree months from time to time. Against this background, the effect of PBCS therapy can be interpreted in such a way that an evolved chronic cluster is first returned to an episodic form followed by ever-increasing pain-free intervals.

\section{Limitations}

This is a retrospective evaluation of $\mathrm{CCH}$ patients treated at our Headache Centre during the last 4 years. Patient 
progressions were described as we observed them under real clinical conditions, a prospective formalization of measurement times and therapy progressions did not take place, likewise naturally no sham or placebo control therapy. In this respect, no efficacy analysis can be derived from the available data as in a controlled study nor can we control any placebo effect. However, the same response patterns across different patients (eg, patient pattern 4, 7) and an effect that persists for many months in some patients (eg, patient 1,2) strongly indicate an intrinsic effect of PBCS therapy. For these reasons, we here limit ourselves to purely descriptive statistics. From what level of pain an attack is evaluated and documented as such is a very subjective decision. However, in so-called "knockknock attacks", in contrast to full-blown attacks, no triptan is taken, so the triptan intake/day helps to differentiate here (see, for example, patient 11). Even if we have not seen any serious undesirable effects, we cannot make a statistically based statement on possible rare side effects due to the small number of cases. However, the safety of the therapy is supported by the fact that the electrical currents used to stimulate the "identified source of the pain" are in the low physiological range and are thus far below the established and clinically classified safe levels delivered by frequency-based stimulation therapies.

\section{Conclusion}

These encouraging cases indicate that PBCS is a promising possibly transformative prophylactic treatment approach for $\mathrm{CCH}$ patients. With PBCS treatment, it could be observed that the $\mathrm{CCH}$ may revert back to an episodic Cluster headache with increasing pain-free intervals. Within the first four treatments, responders can be differentiated from non-responders and show a $60 \%$ reduction in $\mathrm{CH}$ attacks and $80 \%$ reduction in triptan intake. The data support the urgent need for randomized controlled trials for PBCS and should serve as a basis to develop the best-suited inclusion, exclusion criteria, primary endpoints, measurement intervals, and size of such a study.

\section{Abbreviations}

$\mathrm{CH}$, Cluster Headache; $\mathrm{CCH}$, Chronic Cluster Headache; PBCS, Percutaneous Bioelectric Current Stimulation; SPG, Ganglion Sphenopalatineum Stimulation; ONS, Occipitalis Nerve Stimulation; nVNS, noninvasiv Vagus Nerve Stimulation.

\section{Data Sharing Statement}

Further data and patient records, video documentations are presented as supplementary material and are available from the corresponding author on request.

\section{Ethics and Consent Statement}

The PBCS treatment system has been approved and certified for pain treatment by a notified body of the European Commission (CE0482). This study is a retrospective outcome chart analysis of a certified medical device treatment used for individual curative treatments and as such does not require registration nor an ethics committee approval. We obtained written consent to publish patient data and videos from all the respective individuals.

\section{Consent}

Consent for publication has been obtained from all the patients who are shown on the videos.

\section{Author Contributions}

AM treated the patients, collected the data, collected the follow up data and wrote the manuscript. CMC participated in data analysis, contributed to the discussion of the electrophysiological background and contributed to writing and correcting the manuscript. Both authors reviewed and approved the manuscript. All authors contributed to data analysis, drafting or revising the article, gave final approval of the version to be published, and agree to be accountable for all aspects of the work.

\section{Disclosure}

$\mathrm{AM}$ is the founder and medical advisor of the CHP GmbH. He holds several granted and pending patents on the procedure. He receives funding from the German Government for the technical and clinical development of the treatment. The authors report no other conflicts of interest in this work.

\section{References}

1. Rozen TD, Fishman RS. Cluster headache in the United States of America: demographics, clinical characteristics, triggers, suicidality, and personal burden. Headache. 2011. doi:10.1111/j.1526-4610.20 11.02028.x

2. Gaul C, Finken J, Biermann J, et al. Treatment costs and indirect costs of cluster headache: a health economics analysis. Cephalalgia. 2011;31:1664-1672. doi:10.1177/0333102411425866

3. Society, C of T. I. H. The International Classification of Headache Disorders. 3rd ed. Available from: https://ichd-3.org. Accessed March $16,2020$. 
4. Gooriah A, Buture A. Evidence-based treatments for cluster headache. Ther Clin Risk Manag. 2015;11:1687-1696. doi:10.2147/ TCRM.S94193

5. Ashkenazi A, Schwedt T. Cluster headache-acute and prophylactic therapy. Headache. 2011;51:272-286. doi:10.1111/j.1526-4610.2010. 01830.x

6. Magis D, D’Ostilio K, Lisicki M, Lee C, Schoenen J. Anodal frontal tDCS for chronic cluster headache treatment: a proof-of-concept trial targeting the anterior cingulate cortex and searching for nociceptive correlates. $J$ Headache Pain. 2018;19:1-10. doi:10.1186/s10194-018-0904-9

7. Schoenen J, Jensen RH, Lanteri-Minet M, et al. Stimulation of the sphenopalatine ganglion (SPG) for cluster headache treatment. Pathway CH-1: a randomized, sham-controlled study. Cephalalgia. 2013;33:816-830. doi:10.1177/0333102412473667

8. Leone M, Proietti Cecchini A, Messina G, Franzini A. Long-term occipital nerve stimulation for drug-resistant chronic cluster headache. Cephalalgia. 2016;37:1-8.

9. Mueller O, Diener HC, Dammann P, et al. Occipital nerve stimulation for intractable chronic cluster headache or migraine: a critical analysis of direct treatment costs and complications. Cephalalgia. 2013;33:1283-1291. doi:10.1177/0333102413493193

10. Antony AB, Mazzola AJ, Dhaliwal GS, Hunter CW. Neurostimulation for the treatment of chronic head and facial pain: A literature review. Pain Physician. 2019;22:447-477.

11. Halker Singh RB, Ailani J, Robbins MS. Neuromodulation for the acute and preventive therapy of migraine and cluster headache. Headache. 2019;59:33-49. doi:10.1111/head.13586

12. Molsberger A, Mccaig CD. Percutaneous direct current stimulation a new electroceutical solution for severe neurological pain and soft tissue injuries. Med Dev. 2018;11:205-214. doi:10.2147/MDER.S1 63368

13. McCaig CD, Song B, Rajnicek AM. Electrical dimensions in cell science. J Cell Sci. 2009;122(23):4267-4276. doi:10.1242/jcs.023564

14. Mccaig CD, Rajnicek AM, Song B, Zhao M. Controlling cell behavior electrically: current views and future potential. Physiol Rev. 2005;85:943-978. doi:10.1152/physrev.00020.2004

15. Baer ML, Colello RJ. Endogenous bioelectric fields: a putative regulator of wound repair and regeneration in the central nervous system. Neural Regen Res. 2016;11:861-864. doi:10.4103/1673-5374. 184446
16. Baer ML, Henderson SC, Colello RJ. Elucidating the role of injuryinduced electric fields (EFs) in regulating the astrocytic response to injury in the mammalian central nervous system. PLoS One. 2015;10: e0142740. doi:10.1371/journal.pone.0142740

17. Zhao Z, Watt C, Karystinou A, et al. Directed migration of human bone marrow mesenchymal stem cells in a physiological direct current electric field. Eur Cell Mater. 2011;22:344-358. doi:10.22203/ eCM.v022a26

18. Blumenfeld AM, Binder W, Silberstein SD, Blitzer A. Procedures for administering botulinum toxin type A for migraine and tension-type headache. Headache J Head Face Pain. 2003;43:884-891. doi:10.10 46/j.1526-4610.2003.03167.x

19. Molsberger AFF, Boewing G, Diener HC, et al. Designing an acupuncture study: the nationwide, randomized, controlled, german acupuncture trials on migraine and tension-type headache. J Altern Complement Med. 2006;12:237-245. doi:10.1089/acm.2006.12.237

20. Dimitriadou V, Henry B, Brochet B, et al. Cluster headache: ultrastructural evidence for mast cell degranulation and interaction with nerve fibres in the human temporal artery. Cephalalgia. 1990;10:221228. doi:10.1046/j.1468-2982.1990.1005221.x

21. Liberski PP, Academy M, Mirecka B. Mast cells in cluster headache. Ultrastructure, release pattern and possible pathogenetic significance. Cephalalgia. 1984;4:101-106. doi:10.1046/j.1468-2982.1984.0402 101.x

22. Zhu H, Wang X, Huang M, et al. Mast cell activation in the acupoint is important for the electroacupuncture effect against pituitrininduced bradycardia in rabbits. Sci Rep. 2017;7:1-8. doi:10.1038/ s41598-016-0028-x

23. Cunha F, Rajnicek AM, Mccaig CD. Electrical Stimulation Directs Migration, Enhances and Orients Cell Division and Upregulates the Chemokine Receptors CXCR4 and CXCR2 in Endothelial Cells. $J$ Vasc Res. 2019;56:39-53. doi:10.1159/000495311

24. Arnold CE, Rajnicek AM, Hoare JI, et al. Physiological strength electric fields modulate human $\mathrm{T}$ cell activation and polarisation. Sci Rep. 2019;9:1-11. doi:10.1038/s41598-019-53898-5

25. Hoare JI, Rajnicek AM, Mccaig CD, Barker RN, Wilson HM. Electric fields are novel determinants of human macrophage functions. J Leukocyte Biol. 2016;99:1-11.
Journal of Pain Research

\section{Publish your work in this journal}

The Journal of Pain Research is an international, peer reviewed, open access, online journal that welcomes laboratory and clinical findings in the fields of pain research and the prevention and management of pain. Original research, reviews, symposium reports, hypothesis formation and commentaries are all considered for publication. The manuscript management system is completely online and includes a very quick and fair peer-review system, which is all easy to use. Visit http:// www.dovepress.com/testimonials.php to read real quotes from published authors. 